\title{
Plasma Nitriding-Assisted Printing of Stainless Steel Punches for Micro-piercing Process
}

\author{
Tatsuhiko Aizawa $^{1}$ and Tomomi Shiratori ${ }^{2}$ \\ 1. Department of Engineering and Design, Shibaura Institute of Technology, 3-9-14 Shibaura, Minato-City, Tokyo 108-8548, Japan \\ 2. $R$ \& D Center, Komatsu Seiki Kosakusho, Co. Ltd. Shiga-Kuwahara, Ohaza, Suwa-City, Nagano 942-2, Japan
}

\begin{abstract}
MEMS (micro-electric-mechanical-system) required for miniature, thin mechanical parts as a structural member; e.g., the miniature pumping system consisted of ten to twelve thin metallic plates before joining. At present, those thin shaped sheets were fabricated by the chemical etching. Their geometric inaccuracy as well as long leading time often became an engineering issue. Micro-piercing process was expected to make mass production of thin sheet products with complex and accurate geometry for much shorter leading time once the die for this micro-piercing was built in. In the present paper, a new plasma nitriding-assisted printing was proposed as an automatic production line to fabricate the micro-piercing punch. After preparation of CAD-data of the punch head, its two dimensional geometry was ink-jet printed directly on the AISI420 stainless steel die-substrate. The unprinted surface area was only plasma nitrided at $693 \mathrm{~K}$ for $14.4 \mathrm{ks}$ to transform this two dimensional micro-pattern to the three dimensional hardness distribution in the AISI420 substrate. Through the mechanical removal of ink-jet printed area, the flat punch head with sharp edge corners was fabricated in much shorter duration time than the end-milling. SEM-EDX, surface profiling measurement as well as micro-hardness testing were employed to describe each step in the above plasma printing. The thin MEMS stainless steel part with a micro-pendulum as well as three S-letter shaped springs was taken for an example to describe this automatic production procedure of plasma printing from the CAD data mining to the micro-piercing punch finishing.
\end{abstract}

Key words: Plasma printing, ink-jet printing, plasma nitriding, sand-blasting, micro-piercing, stainless steel punch.

\section{Introduction}

Micro-stamping has grown up to be popular among several methods to fabricate the miniature, thin metallic parts and members [1]. In fact, thin orifice plates with the thickness of $0.1 \mathrm{~mm}$ were precisely micro-pierced to have one to sixteen small through-holes with the diameter of 80 to $100 \mu \mathrm{m}$ for regular fuel injection in safety [2]. Those micro-piercing punches often suffered from the shortage of tool life; high qualification must be equipped to the punch materials. Micro-pumping for medical treatment unit was a typical MEMS, which consisted of ten to twelve thin stainless steel parts. Their geometric configuration must be precisely pierced into each constituent sheet before joining them

Corresponding author: Tatsuhiko Aizawa, PhD, professor, research fields: materials science engineering, metal forming, micro-manufacturing to a product [3]. At present, the chemical etching was mainly used to make complex shaping into metallic sheets. To save the dimensional irregularity of the through-hole walls in this chemical etching and to be free from the instability of aged chemical solution, more reliable micro-piercing process is expected as an alternative method. In the above applications, how to build up the micro-punches with sufficient toughness and geometric accuracy must be a key to solve the engineering issues.

Micro-milling [4], micro-EDM (electric discharge-machining) [5] and laser machining [6] were reliable means to fabricate these micro-punches. However, with reduction of the product size, the leading time for production significantly increased in those traditional approaches. Besides for the exponentially increasing tooling time, huge labor cost was also needed to prepare for the CAM data before tooling. Tooling as well as CAM data mining hinder 
the automatic production stream from the CAD data to the micro-piercing punch.

The plasma assisted micro-patterning method was first proposed in Ref. [7] with use of PECVD (plasma enhanced chemical vapor deposition) nitriding at 673 $\mathrm{K}$ at 2 to $3 \mathrm{~Pa}$. Even by using the ceramic masking technique, the nitriding front end advanced even below the masked area so that the masking pattern never transformed to the nitrided textures. The authors succeeded in the micro-texturing into the stainless steel substrates with use of the high density plasma nitriding at $693 \mathrm{~K}$ at 30 to $100 \mathrm{~Pa}$ [8]. First, both convex and concave micro-dimples were precisely drawn as a two-dimensional micro-pattern onto the stainless steel mold surface by using the metallic masks $[9,10]$, or, by the ink-jet printing [11]. After plasma nitriding and mechanical blasting, the mold unit for cassette die system was successfully fabricated so that the original two dimensional micro-dimples were duplicated as micro-disc textures on the plastic product surfaces through the injection molding $[9,10]$. This plasma printing was also applied in trial to fabrication of the micro-piercing punches and dies [12].

In the present paper, this plasma nitriding assisted printing is redesigned and used as an automatic production tool to fabricate the miniature punches for micro-piercing the stainless sheet with the circular micro-pendulum and three S-letter shaped springs. The stainless steel substrate with the size of $5 \times 5 \mathrm{~mm}^{2}$ is prepared for plasma printing. The micro-punch geometry is designed to have three sectioned heads to form the circular micro-pendulum part with three S-letter shaped springs formed into the sheet. First, the original two dimensional micro-pattern for this micro-piercing punch head is ink-jet printed directly from the CAD data onto the substrate. Through the plasma nitriding, the unprinted, bare substrate surface is selectively nitrided to have much higher hardness than the printed surfaces with matrix hardness. The sand-blasting is utilized to fabricate the micro-punch by mechanical removal of low hardness area. Without use of mechanical milling, micro-EDM or chemical etching, the micro-piecing punch is automatically fabricated from its CAD data. Through the precise observation and measurement, the role of plasma nitriding in this plasma printing process is understood with description on the relationship between the selectively nitriding process and the dimensional accuracy of micro-punch geometry. The superiority of this plasma printing to the end-milling is discussed through comparison of the leading time between two methods.

\section{Experimental Procedure}

\subsection{Plasma Nitriding Assisted Printing}

This plasma nitriding assisted printing process consists of three steps: (1) Ink-jet printing to prepare for the initial two dimensional micro-patterns, (2) Selective nitriding and hardening of the unprinted surfaces, and (3) Selective mechanical removal of the printed soft area. These three steps are explained as an automatic production line to fabricate the micro-piercing punch, as depicted in Fig. 1. Table 1 lists the experimental set-ups and conditions for these three steps.

In the first step, the original CAD data of the micro-punch shape are exported into the ink-jet printer to build up the series of commands to draw the initial pattern onto the punch substrate. Fig. 1a illustrates the printed image of micro-piercing punch head by using its CAD data. The white area corresponds to the punch head; the black area is free from piercing and left as a metallic sheet product with the circular pendulum and three S-letter shaped springs. In practical operation, the initial CAD data, which are represented by Fig. 1a, are first transferred to the command file through the software of "Illustrator". The flat bed type ink-jet printer (Mimaki, Co., Ltd.) is used to draw the negative micro-pattern of piercing punch, which is represented by the black area in Fig. 1a. This drawn pattern must preserve during the plasma nitriding at the elevated temperature. After preliminary experiments [12, 13], 
a)

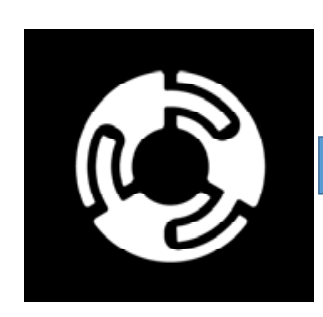

Ink-jet printing of punch head shape
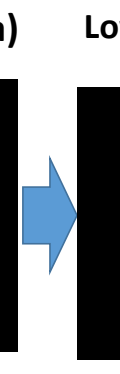

b)

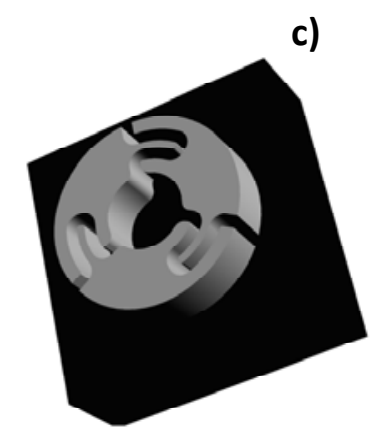

Mechanical removal of initially printed area

Fig. 1 Three steps of the plasma nitriding assisted printing. a) Ink-jet printing, b) Low-temperature plasma nitriding, and c) Mechanical removal.

Table 1 The plasma printing procedure and process conditions in the present experiment.

\begin{tabular}{|l|l|l|}
$\begin{array}{l}\text { Steps in the } \\
\text { Plasma Printing }\end{array}$ & $\begin{array}{l}\text { Experimental } \\
\text { Set-up }\end{array}$ & $\begin{array}{l}\text { Experimental } \\
\text { Conditions }\end{array}$ \\
\hline Ink-Jet Printing & $\begin{array}{l}\text { /Spatial resolution: } \\
1 \mu \mathrm{m} \\
\text { /Raster scanning } \\
\text { /Ink: Carbon black } \\
\text { and primer }\end{array}$ \\
\hline Plasma Nitriding & $\begin{array}{l}\text { /RF-DC plasmas } \\
\text { /Automatic input- } \\
\text { output matching } \\
\text { / }\end{array}$ \\
/Automatic control \\
Sand Blasting
\end{tabular}

this ink was composed of the polymer primer and the carbon black by the ratio of $50 \%$ to $50 \%$. In addition, the as-printed pattern on the substrate was further heat-treated to improve its heat resistance.

The high density plasma nitriding system is used to make selective nitriding and hardening on the white areas in Fig. 1b. Since the black area in Fig. 1b is free from the nitriding, it is never hardened and it remains to have the bare substrate hardness. In case of the martensitic stainless steel without preliminary heat treatment, the hardness of black area remains to be $250 \mathrm{HV}$ even after the plasma nitriding. Through this nitriding process, the printed micro-pattern in Fig. 1a transforms to the hardness depth profile with high hardness in the white area and low hardness in the black area. In the following experiments, the nitriding parameters as listed in Table 1 are employed as a standard condition. 
The sand-blasting system (Fuji-Seisakujyo, Co. Ltd.) is also employed to make selective removal of the relatively soft materials from substrate. Its capability of mechanical removal depends on the blasting media as well as the hardness of nitrided area. When using the silica particles with the average diameter of $30 \mu \mathrm{m}$, the unprinted areas with the hardness higher than $800 \mathrm{HV}$ are free from blasting; the printed black areas in Fig. 1a are selectively removed in a few minutes to form the micro-piercing punch in Fig. 1c, the head of which corresponds to the white area in Figs. 1a and 1b, respectively.

\subsection{Specimens}

AISI420 stainless steel substrate with the diameter of $25 \mathrm{~mm}$ and the thickness of $5 \mathrm{~mm}$ was mirror-polished in its either surface.

\subsection{Observation and Measurement}

SEM (scanning electron microscope) as well as EDX (electron diffraction x-ray) were employed to make observation and nitrogen element mapping on the cross-section of die-substrate. The micro-Vickers testing (Mitsutoyo, Co. Ltd.) was utilized to measure the hardness depth profile. Three dimensional profilomer (Keyence, Co. Ltd.) was also used to measure the depth profile and the peak-to-valley distance of a single micro-piercing punch as well as an assembly of micro-punches.

\section{Results and Discussion}

The plasma printing procedure is explained as an automatic production line from the CAD-data mining to the micro-piercing punch finishing. The role of low temperature plasma nitriding process in this line is further described to demonstrate the selective nitriding and hardening of unprinted surface of substrate in the plasma printing. The assembly of micro-piercing punches are fabricated by the same procedure as needed for a single punch.

\subsection{Automatic Procedure from CAD-data to Micro-piercing Punch Finishing}

The actual production line for fabrication of the micro-piercing punch to form the circular pendulum as well as three S-letter shaped springs into the thin metallic sheet is explained in correspondence to the production-line design in Fig. 1.

At the first step, the initial two dimensional micro-pattern for this micro-piercing punch is directly ink-jet printed onto the polished surface of AISI420 substrate. The flat-bed type dispenser was utilized to make ink-jet printing in the raster scanning manner. Fig. 2a depicts the ink-jet printed pattern drawn on the AISI420 substrate. In correspondence to the black area in Fig. 1a, the ink is line-drawn into the inside of white-lined contours, besides for the outer black area. The circular pendulum as well as three S-letter shaped spring parts are accurately drawn with the tolerance of 5 to $10 \mu \mathrm{m}$ in width. Owing to the optimum composition of ink and its post-treatment, these printed patterns remain as they were, even after the following plasma nitriding.

In the second step, the high density plasma nitriding system was employed to make selective hardening of the unprinted surface at $693 \mathrm{~K}$ for $14.4 \mathrm{ks}$ at $70 \mathrm{~Pa}$. During the nitriding process, both the temperature and pressure were automatically controlled to be constant within the tolerance of $1 \mathrm{~K}$ and $0.1 \mathrm{~Pa}$, respectively. The flow rate of nitrogen and hydrogen gases was also controlled to be $150 \mathrm{ml} / \mathrm{min}$ and $30 \mathrm{ml} / \mathrm{min}$, respectively. The plasma nitrided AISI420 substrate is shown in Fig. $2 \mathrm{~b}$ together with the measured hardness at each position on the punch head. This punch head area or the unprinted area has higher micro-Vickers hardness than $850 \mathrm{HV}$; other areas or printed areas by the ink-jet printing have only $250 \mathrm{HV}$, equivalent to the matrix hardness of bare AISI420 specimen.

The sand-blasting was employed as the third step to mechanically remove the softer area of AISI420 specimen. After the preliminary study [13], the surface area with lower hardness than $800 \mathrm{HV}$ was possible to 
be removed by the present sand-blasting conditions in Table 1. Fig. 2c depicts the surface profile of micro-piercing punch after sand-blasting for 5 minutes. The ink-jet printed area of AISI420 substrate is removed to become a bottom of punch head. Three sectors, which are not masked by the ink-jet printing, turn to be a punch head with the height of $50 \mu \mathrm{m}$. This selective removal of un-hardened or un-nitrided surface areas forms the accurate three dimensional shape of micro-piercing punches. Fig. 3 shows the cross-section of formed punch head across the line A in Fig. 2c. The punch head remains to be flat since the original mirror-polished surface is preserved even in nitriding. In addition, the sharp edge of punch is formed by the significant difference in nitrogen concentration, where no nitrogen atoms diffuse in the printed area but they
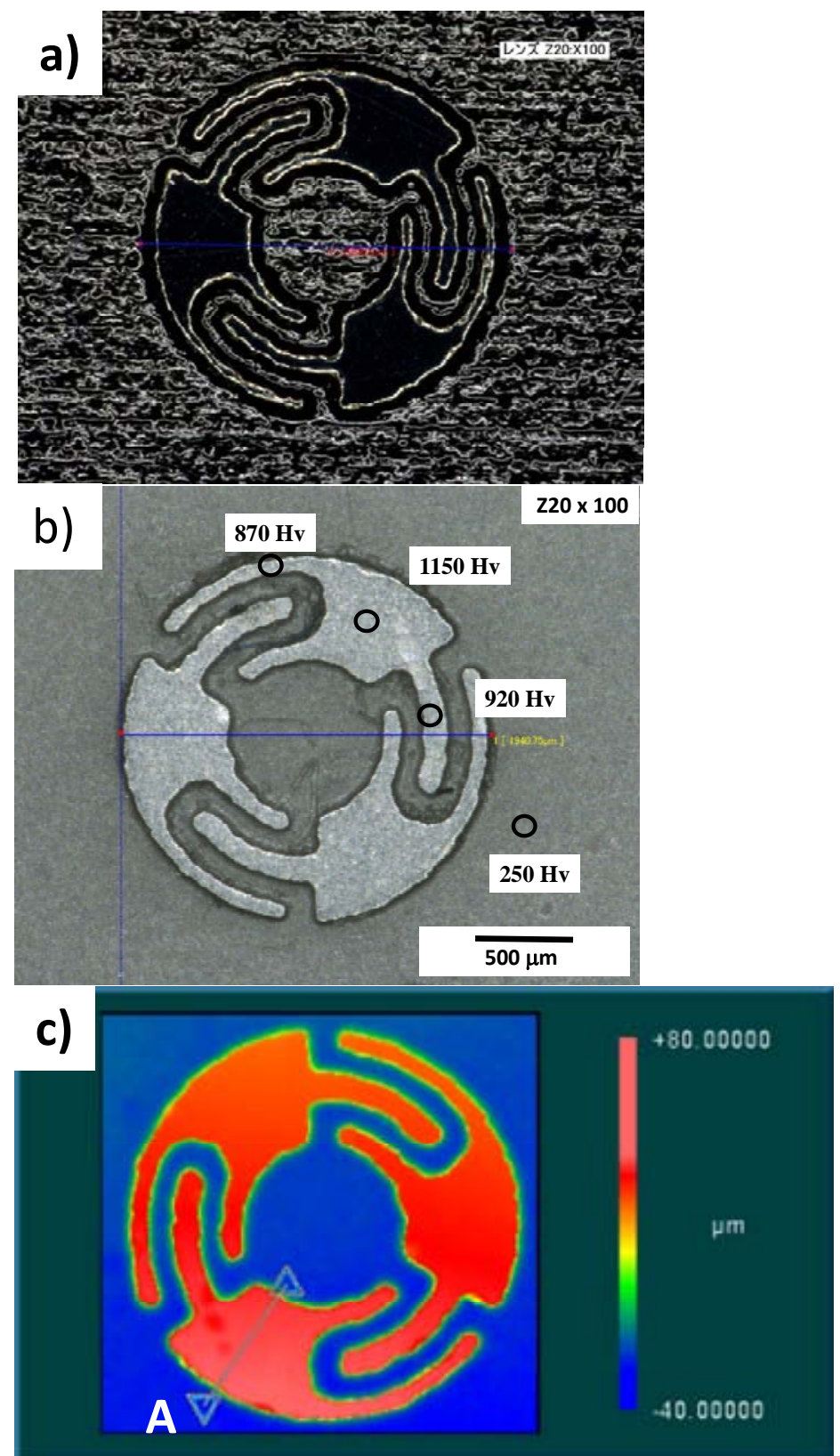

Fig. 2 Plasma printing of the piercing punch for circular pendulum with three S-letter shaped springs into AISI420 die specimen. a) The initial micro-pattern drawn onto the surface of die specimen by the ink-jet printing, b) The plasma nitrided specimen together with the measured hardness, and, 3) The finished punch head after sand-blasting. 
are present only in the un-printed one. The measured sharp edge angle in normal from Fig. 6 is estimated to be $86^{\circ}$ to $87^{\circ}$.

\subsection{Role of Plasma Nitriding in the Plasma Printing}

The plasma nitriding process plays a key role in this plasma printing; Two dimensionally drawn pattern on the AISI420 substrate automatically transforms to hardness profiled die by this low temperature plasma nitriding. In order to construct this automatic production line from the bare substrate to the net-shaped punch, the unprinted area by the ink-jet printing must be selectively hardened by this low temperature plasma nitriding before selective removal of initially micro-patterned, softer area by sand-blasting. As surveyed in Ref. [14], in the commercial high temperature plasma nitriding processes, the iron and chromium nitrides are formed as a precipitate to strengthen the stainless steel matrix by plasma nitriding when the holding temperature exceeds $750 \mathrm{~K}$. Besides for the general difficulties in processing at the elevated temperature, formation of the brittle white layer has a risk to lower the surface quality. Lowering this holding temperature down to be less than $700 \mathrm{~K}$, the infiltrating nitrogen interstitials during the plasma nitriding are present as a solute in the extended austenitic stainless steels or $\gamma(\mathrm{N})$ for AISI316/304 stainless steels [15], or, as a solute in the extended martensitic ones or $\alpha^{\prime}(\mathrm{N})$ for AISI420 stainless steels [8]. Under this processing, the unprinted substrate surface is expected to be hardened by nitrogen solid solution.

After SEM and EDX analysis on the cross-section of the nitrided AISI420 substrate, the micro-structure of nitrided part in the substrate is described together with the nitrogen content distribution. Fig. 4 shows the micro-structure of nitrided area as well as the nitrogen mapping at the vicinity of the border between the unprinted and the printed soft areas. Little nitrogen atoms are detected below the printed pattern; while the nitrided depth profile extends from the edge of border to the un-printed area. That is, the printed surface is

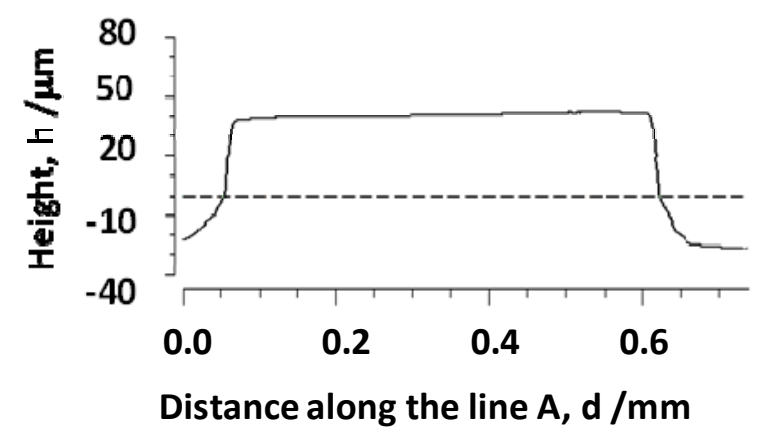

Fig. 3 Cross-section of the formed punch head across the line A in Fig. 2 c).

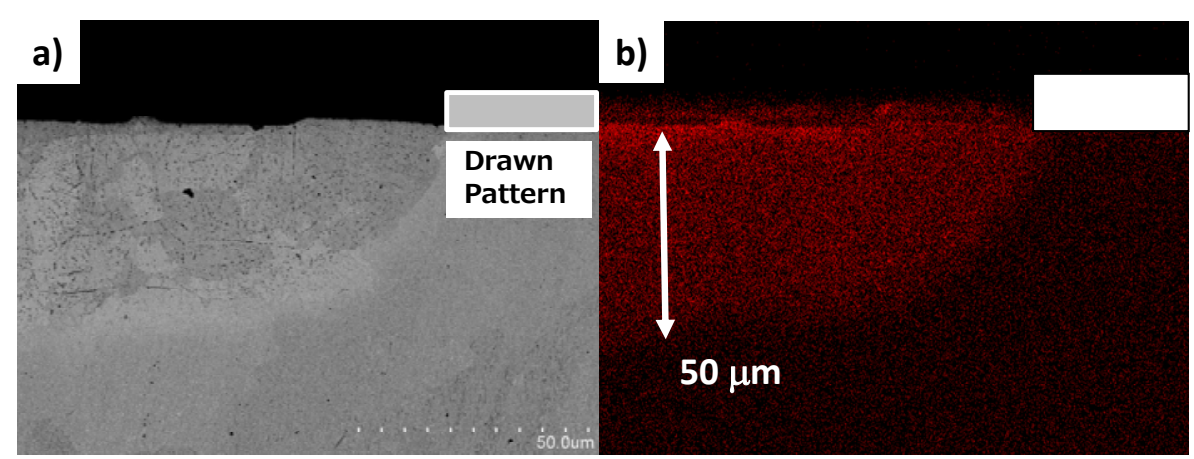

Fig. 4 Selective nitriding process into the depth of AISI420 specimen only on the unprinted area. a) Cross-sectional SEM image at the vicinity of the border between the printed and unprinted surfaces, and b) Nitrogen mapping by EDX. 
never nitrided; while the nitrided layer depth reaches to $50 \mu \mathrm{m}$ in the unprinted surface. This selective nitriding only into the unprinted substrate is thought to drive its selective hardening through high nitrogen concentration from its surface to depth only in the unprinted area. In order to investigate the relationship between the nitrogen solute distribution in depth and the hardness depth profile, the nitrogen content is measured from the unprinted surface to the depth in every $50 \mu \mathrm{m}$ by EDX. Fig. 5 shows the nitrogen concentration across the nitrided layer thickness of unprinted area. At the vicinity of nitrided AISI420 substrate surface, the nitrogen content becomes maximum by 10 mass\%. The nitrogen content gradually decreases in depth; it forms a plateau where the nitrogen content becomes nearly constant by 4 mass $\%$. This plateau is preserved in depth just before approaching to the nitriding front end at the depth of $50 \mathrm{~m}$. Fig. 6 depicts the distribution of measured hardness from the surface to the nitriding front end. Higher hardness than 1,000 $\mathrm{HV}$ is attained from the vicinity of surface towards the end of plateau. In the depth beyond the nitriding front end, the hardness is reduced to the matrix hardness of $250 \mathrm{HV}$ for the bare AISI420.

This selective nitriding and hardening becomes a key to make hardening of the unprinted substrate surface and to construct the micro-textured punch and die by the mechanical removal process. How deep the micro-textures can be cut into the depth of punch and die by this plasma printing, depends on the nitrogen solute distribution as well as the nitrided layer thickness. The relation between the punch height and the nitrided layer thickness is investigated in the simultaneous fabrication of nine punches from the same CAD-data.

\subsection{Simultaneous Fabrication of Multi-punches}

Within the spatial resolution by the ink-jet printing, various stainless steel punch heads with the tailored geometry are produced only by updating the initial CAD data. An assembly of micro-piercing punches or multi-punch heads are also fabricated without significant increase of processing time. Nine micro-piercing punches are simultaneously formed by the present plasma printing. Fig. 7a depicts the nine initial micro-patterns, the each of which is drawn by the same procedure as used for ink-jet printing of a single micro-piercing punch as seen in Fig. 2. Through duplication of the raw CAD data for a single punch in Fig. 2a, the controlling commands to drive the ink-jet printer are easy to be programmed for drawing these

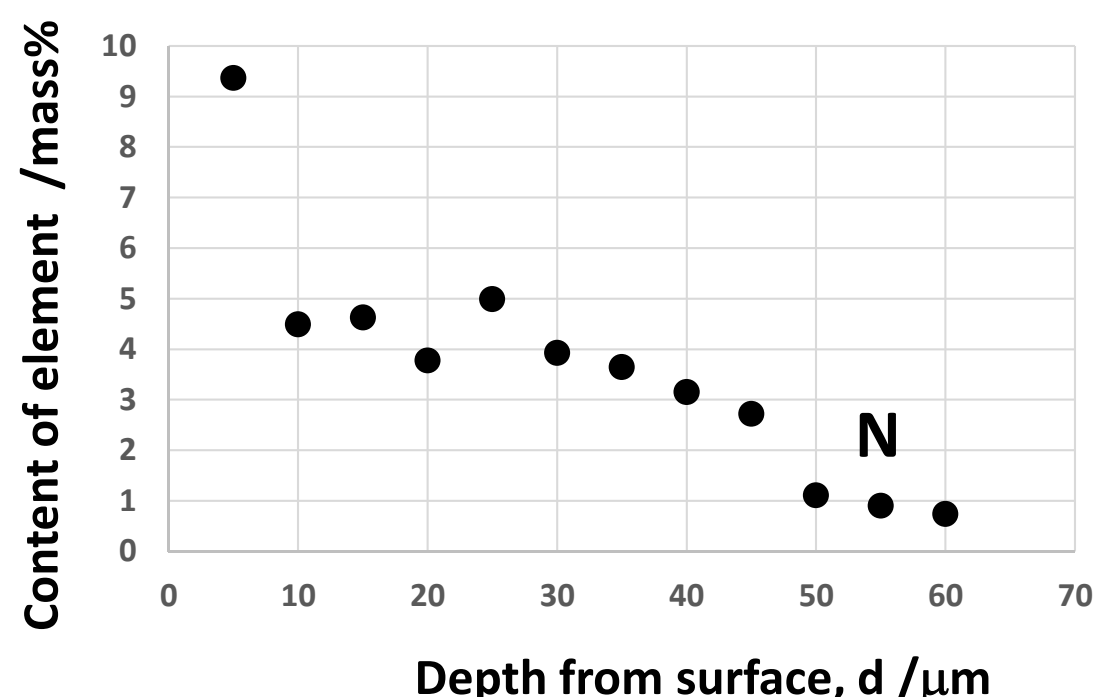

Fig. 5 Nitrogen concentration from the surface to the depth, measured by EDX, in the unprinted area. 


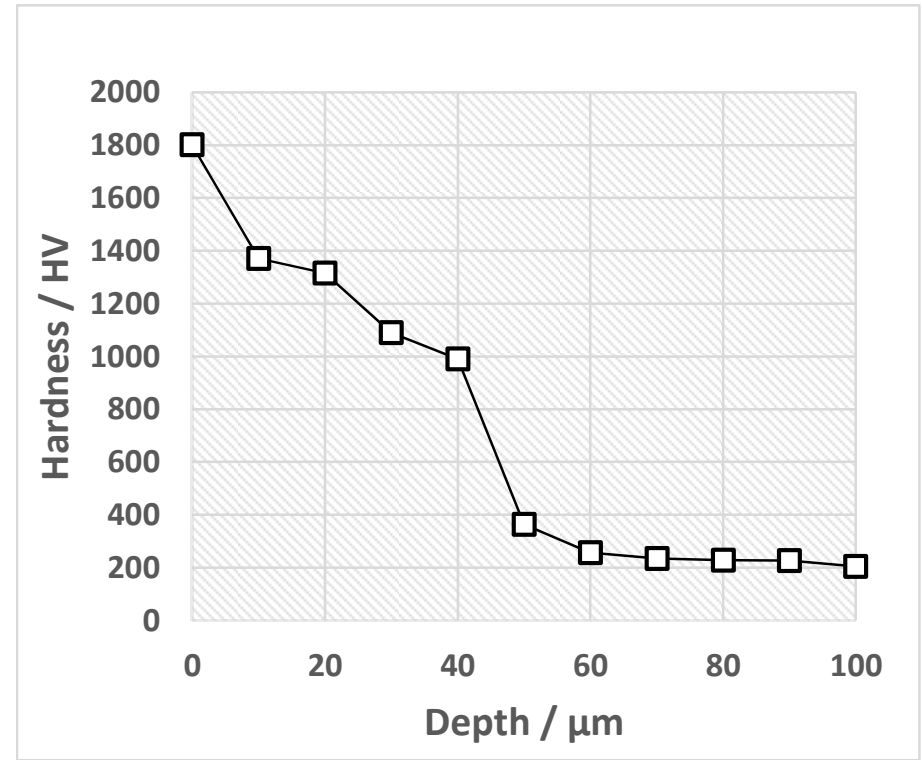

Fig. 6 Hardness depth profile from the surface to the depth in the unprinted area.

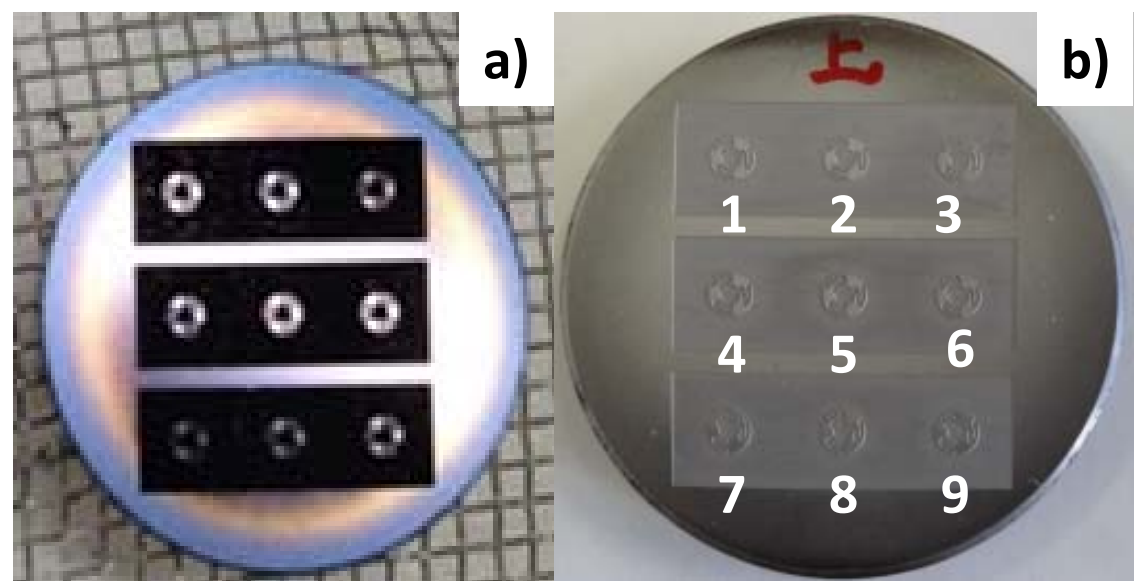

Fig. 7 Simultaneous fabrication of nine micro-punch heads by the plasma printing. a) Nine punch head patterns drawn on the substrate by the ink-jet printing, and, b) Nine punch heads fabricated by the plasma printing.

nine punch geometries onto the substrate. There is no change even in other steps of plasma printing between single punch and multi-punch fabrications. Without significant increase of production cost, nine micro-piercing punches are formed on the same substrate as shown in Fig. 7b. This plasma printing has flexibility enough to fabricate the hundreds of micro-punch units simultaneously.

The peak-to-valley (PV) height of each punch head in Fig. $7 \mathrm{~b}$ is measured to calculate the average PV height for nine punches and its deviation. Fig. 8 depicts the statistical distribution of measured PV heights for nine punch heads. The average PV height is $50 \mu \mathrm{m}$, and its deviation, 15 to $20 \mu \mathrm{m}$. This deviation in the measured PV heights might be attributed to manual operation to keep the shooting angle against the silica powders by $60^{\circ}$ in the sand-blasting step. This average is just corresponding to the nitrided layer thickness of $50 \mu \mathrm{m}$, in Figs. 4 and 5, respectively. The PV height of single-punch in Fig. 3 as well as the average PV height of nine punches in Fig. 8 are nearly equal to the nitrided layer thickness, $50 \mu \mathrm{m}$, in Fig. 4. The printed or un-nitrided, soft surface area is selectively removed by the sand-blasting homogeneously so that the nitrided and hardened surfaces are left as a punch head. Then, the micro-piercing punch head 


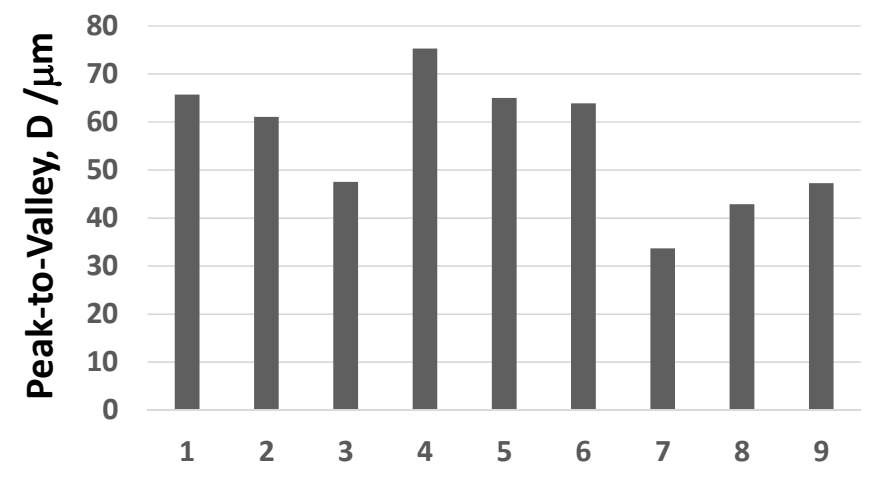

Number of specimen in Fig. 7

Fig. 8 The statistic distributions of measured peak-to-valley heights for nine micro-piercing punches simultaneously fabricated by the same plasma printing process.

Table 2 Processing time necessary for each step in the plasma printing.

\begin{tabular}{|l|c|}
\hline Step in the plasma printing & Production time \\
\hline $\begin{array}{l}\text { Ink-jet printing } \\
\text { including the set-up }\end{array}$ & $1.2 \mathrm{ks}$ \\
\hline Plasma nitriding & $14.4 \mathrm{ks}$ \\
\hline Sand blasting & $0.3 \mathrm{ks}$ \\
\hline
\end{tabular}

by the plasma printing has nearly the same height as the nitrided layer thickness. The border between the printed and unprinted areas forms the sharp edge of micro-piercing punch heads, as depicted in Fig. 2c and Fig. 3, respectively.

\subsection{Evaluation on the Production Cost}

The production cost is compared between the micro-milling and the present plasma printing in this formation of micro-punch with $5 \times 5 \mathrm{~mm}^{2}$ as shown in Fig. 2c. In case of the normal micro-milling of prehardened AISI 420 substrate with 1,000 HV, the feeding depth $\left(V_{f}\right)$ as well the cutting speed $\left(V_{m}\right)$ must be reduced as much as possible even by using the hard-coated fine cutting tools; e.g., $V_{f}=10 \mu \mathrm{m}$ and $V_{m}$ $=50 \mu \mathrm{m} / \mathrm{s}$ by using the fine tool with the diameter of $10 \mu \mathrm{m}$. The black area in Fig. 1a or the printed area in Fig. 2a is mechanically removed down to the depth of $50 \mu \mathrm{m}$. The average cutting pass width is assumed to be $60 \mu \mathrm{m}$ in Fig. 2a; the cutting length to build up three-sectioned punch head corresponding to the circular pendulum as well as three S-letter shaped spring parts, is estimated to $2,500 \mathrm{~mm}$. Then, the total leading time reaches to $(2,500 \mathrm{~mm} / 50 \mu \mathrm{m} / \mathrm{s}) \times(50 \mu \mathrm{m}$ $/ 10 \mu \mathrm{m})=250 \mathrm{ks}$ without exchange of tools. Table 2 summarizes the production time for each step in the plasma printing. The total time is $15.9 \mathrm{ks}$. Except for time consuming work for preparation of CAM data, the production cost is reduced by $1 / 15$ by the present plasma printing to form a single micro-piercing punch. As before mentioned, this automatic production line to build up a single micro-punch is also true to simultaneous fabrication process to make multi-punches as an assembly with $\mathrm{N}$ punch units. On the other hand, the micro-milling requires $250 \times \mathrm{N}$ ks only for actual milling process.

The same procedure as used for fabrication of the micro-piercing punch in this study is also employed to produce the core die with sufficient compatibility to the punch in geometry and dimension. The punch head was produced from the white area in Fig. 1a by removing the black one. This initial micro-pattern to 
be drawn in Fig. 1a or Fig. 2a is modified by changing the black area to the white one and reflecting this image. Then, the starting micro-pattern to draw the core-die surface is prepared automatically. Under the present automatic production line, both the punch and core-die, or, both the punch and core-die assemblies are also fabricated as a die-set.

\section{Conclusion}

The micro-piercing punch with the head size of $5 \times$ $5 \mathrm{~mm}^{2}$ was fabricated by the plasma printing method. This automatic production lines consisted of three steps. The initial two dimensional pattern for this miniature punch was ink-jet printed onto the surface of AISI420 stainless steel substrate. Through the plasma nitriding at $693 \mathrm{~K}$ for $14.4 \mathrm{ks}$ by $70 \mathrm{~Pa}$, this drawn micro-pattern was transformed into the three dimensional hardness depth profile. The diffusing nitrogen content discontinuously distributed across the border between the bare AISI40 surface and the masked area by printing. No nitrogen atoms were strictly present below the printed surface. This selective nitriding resulted in the selective hardening in this process. The printed area had bare hardness of AISI420 stainless steel by $250 \mathrm{HV}$ while the unprinted area, more than 1,000 HV. This hardness profile even in depth changed itself to the three dimensional punch geometry by selectively removing the initially drawn, un-nitrided micro-patterns.

The sharp-edge corner required for a piercing punch was preserved by the significant hardness change across the border between the initially printed area and the un-printed one. The hardness necessary for a punch head was attained by selective hardening of unprinted area since the nitrogen atoms diffused into the depth of AISI420 only through its unprinted surface.

The punch head was accurately shaped by sand-blasting in fairly good agreement to the initially drawn micro-patterns on the AISI420 substrate. The punch height after finishing during the sand-blasting was nearly equal to the nitrided layer thickness. Furthermore, the leading time for tooling was much saved by this approach to reduce the production cost.

In this automatic production line, the finished micro-punch and die are fabricated into the steel substrate only by preparing the CAD-data for tooling. Their top surface geometry is determined by the micro-patterning via the ink-jet printing; their side surfaces are formed by sand-blasting down to the nitrided layer thickness. During nearly the same processing time as needed for production of a single micro-punch with the complex shape, the assembly of multi-punch heads are simultaneously fabricated as a special tool for micro-piercing the multi-holes.

In the present plasma printing, the sand-blasting was employed to form the punch head and edges. The chemical etching can be utilized to make full use of the difference in the nitrogen contents between the printed and the unprinted areas and to increase the spatial resolution in formation of punch heads. Furthermore, this plasma printing can be applied to the curved surface of milling rolls and ball indenters. Once the initial micro-patterns are ink-jet printed onto the curved roll and ball surfaces, the tools for precise shape-rolling and indention are automatically fabricated in the similar procedure.

\section{Acknowledgements}

The authors would like to express their gratitude to Mr. T. Takashima, Mr. S. Kurozumi (SIT) and Mr. Y. Suzuki (Komatsu Seiki Kosakusho, Co. Ltd.) for their help in experiments. This project is financially supported in part by METI and MEXT projects, respectively.

\section{References}

[1] Vollertsen, F., Biermann, D., Hansen, H. N., Jawahir, I. S., and Kuzman, K. 2009. "Size Effects in Manufacturing of Metallic Components.” CIRP Ann-Manuf. Technol 58: 566-87.

[2] Komatsu, T., Kobayashi, H., Torizuka, S., and Nagayama, S. 2012. "Micro Hole Piercing for Ultrafine Grained Steel.” J. Autom. Tech. 6 (6): 802-6. 
[3] Yang, M., Kabasawa, Y., and Ito, K. 2009. "Fabrication of Micro Metallic Valve and Pump.” In Proceedings of Fourth International Conference on Experimental Mechanics.

[4] Denkena, B., Koehler, J., and Laestner, J. 2012. "Efficient Machining of Micro-dimples for Friction Reduction.” In Proc. of 7th ICOMM, 85-9.

[5] Jiang, Y., Zhao, W. S., Kang, X. M., and Gu, L. 2011. "Adaptive Control for Micro-hole EDM Process with Wavelet Transform Detecting Method.” In Proc. of 6th ICOMM, 207-11.

[6] Etsion, I. 2004. "Improving Tribological Performance of Mechanical Components by Laser Surface Texturing.” Tribology Letters 17 (4): 733-7.

[7] Marcos, G., Guilet, S., Cleymand, F., Thiriet, T., and Czerwiec, T. 2011. "Stainless Steel Patterning by Combination of Micro-patterning and Driven Strain Produced Plasma Assisted Nitriding.” Surf. Coat. Technol. 205: 5275-9.

[8] Santojojo, D., Aizawa, T., Muraishi, S., and Morita, H. 2014. "Micro-texturing of Stainless Steels via High Density Plasma Nitriding.” In Proc. of 9th ICOMM, 90: $1-8$.
[9] Aizawa, T., and Yamaguchi, T. 2015. "Plasma Nitriding Assisted Micro-Texturing into Martensitic Stainless Steel Molds for Injection Molding." In Proceedings of the 4M/ICOMM2015, 454-459.

[10] Aizawa, T., Suga, H., and Yamaguchi, T. 2015. "Plasma-Nitriding Assisted Micro-Texturing into Stainless Steel Molds.” In Proc. of 4th ICNT, 9002/1-6.

[11] Aizawa, T., Takashima, T., and Shiratori, T. 2015. "Plasma Printing to Fabricate the Micro-piercing Dies for Miniature Metal Products.” In Proc. of 8th AWMFT, October, Suwa, Japan 1: 1-6.

[12] Aizawa, T., Takashima, T., Shiratori, T., and Suzuki, Y. 2016. "Fabrication of Micro-piercing Dies and Punches via Plasma Printing.” In Proc. of 11th ICOMM, March, 2: 1-4.

[13] Aizawa, T., Suga, H., Katoh, T., Silva, L.U., and Yamaguchi, T. 2015. "Plasma Nitriding Assisted Micro-texturing.” Res. Rep. SIT59-1: 9-18.

[14] Aizawa, T., and Sugita, Y. 2013. "High Density RF-DC Plasma Nitriding of Steels for Die and Mold Technologies.” Res. Rep. SIT57-1: 1-10.

[15] Anzai, M. 2009. Surface Treatment for of Dies and Molds. Nikkan Kogyo Shimbun, Japan. 